\title{
SECONDARY ELECTRON EMISSION PROPERTIES
}

\author{
by J.J. SCHOLTZ, D. DIJKKAMP and R.W.A. SCHMITZ
}

Philips Research Laboratories, Prof. Holstlaan 4, 5656 AA Eindhoven, The Netherlands

\begin{abstract}
In this paper an introduction is given to secondary electron emission properties. It is shown that the reduced secondary emission yield $\delta / \delta_{\mathrm{m}}$ as a function of the reduced primary energy $E_{\mathrm{p}} / E_{\mathrm{m}}$ can be described by a universal curve. It is found that it is easier to use the measurement of the maximum secondary electron emission $\delta_{\mathrm{m}}$ and the energy $E_{\mathrm{m}}$ at which this maximum is reached to determine the suitability of a coating for use in the display than direct measurement of the first crossover energy $E_{\mathrm{l}}$. The value of $\delta_{\mathrm{m}}$ and $E_{\mathrm{m}}$ can be used to derive $E_{\mathrm{I}}$. Furthermore, it is observed that in any material the elastic fraction of the secondary electrons exhibits a universal behaviour as a function of $E_{\mathrm{p}}$. Fits to $\delta\left(E_{\mathrm{p}}\right)$ and the energy distribution of the secondary electrons are proposed which can be used in Monte Carlo simulations.
\end{abstract}

Keywords: secondary electron emission, flat and slim display, energy distribution, Zeus display, $\mathrm{MgO}$.

\section{Introduction}

For the Zeus display knowledge of the mechanism of the transport of electrons along the surface of insulators by means of 'hopping' is very important $[1,2]$. The hopping behaviour is mainly determined by the secondary emission parameters of the insulator on which this hopping takes place. Knowledge of these secondary emission parameters is necessary for determining its suitability for use in the display.

Secondary emission takes place when a material is bombarded by electrons, ions or photons. In our case we are only interested in the effect of bombardment by electrons. The secondary electron emission yield $\delta$ is defined as:

$$
\delta=\frac{i_{\mathrm{s}}}{i_{\mathrm{p}}},
$$


where $i_{\mathrm{p}}$ is the primary current of the impinging electrons and $i_{\mathrm{s}}$ is the current of all electrons leaving the surface, so including both the elastically and inelastically scattered electrons. This is different from definitions used in literature, where $i_{\mathrm{s}}$ often is defined as the current of the 'true' secondaries. 'True' secondaries are frequently defined as the secondaries with an energy lower than $50 \mathrm{eV}$. A typical $\delta$-curve, i.e. $\delta$ as a function of the primary energy $E_{\mathrm{p}}$, and a typical $N\left(E_{\mathrm{s}}\right)$ curve, i.e. the distribution of the secondary electron energy $E_{\mathrm{s}}$, are shown in Fig. 1.

As shown in Fig. 1, $\delta$ increases as a function of $E_{\mathrm{p}}$ at very low primary energy. This is a result of the fact that with increasing beam energy more secondary electrons can be excited. However, secondary electrons have a very small escape depth due to their low energy $(0$ to $50 \mathrm{eV})$. This escape depth is in the order of $10 \mathrm{~nm}$ [3]. At a certain beam energy the penetration depth of the primary electrons becomes higher than the escape depth of the secondaries, resulting in a decrease of $\delta$. From Fig. la it is observed that $\delta=1$ for two primary energies. These energies are denoted as the first (low energy) and second (high energy) crossover energies $E_{\mathrm{I}}$ and $E_{\mathrm{II}}$.

For the Zeus display especially the first crossover point $E_{\mathrm{I}}$ and the average kinetic energy of the secondary electrons $E_{\mathrm{a}}$ are of importance. These parameters are of major influence on the electric field (the 'transport field') needed to transport electrons along the channels by means of the hopping mechanism $[1,2]$. In general it is found that a small value for $E_{1}$ results in a small value for the minimal transport field. $\mathrm{MgO}$ is used in the display, because of its small value for $E_{\mathrm{I}}$ [4] and its stability under electron bombardment [5].

In Sec. 2 of this paper we show how secondary electron emission can be understood in a more quantitative way. In Sec. 3 we describe the experimental set-up used for determining $\delta\left(E_{\mathrm{p}}\right)$ and $N\left(E_{\mathrm{s}}\right)$. In Sec. 4 we show and discuss measurements of $\delta\left(E_{\mathrm{p}}\right)$ for several materials, illustrating the universal behaviour of the reduced yield curve. Furthermore, we show measurements of the first crossover energy $E_{\mathrm{I}}$ and finally we will discuss measurements on $N\left(E_{\mathrm{s}}\right)$.

\section{Semi-empirical theory}

To obtain a better insight into the mechanisms responsible for the secondary electron cmission, wc give a concisc description of the semi-empirical theory developed by Dionne [6]. In deriving $\delta\left(E_{\mathrm{p}}\right)$ Dionne makes the following assumptions:

(1) The number of secondaries produced per unit length at position $x$ (measured along the path of the primary electron) is equal to the energy 

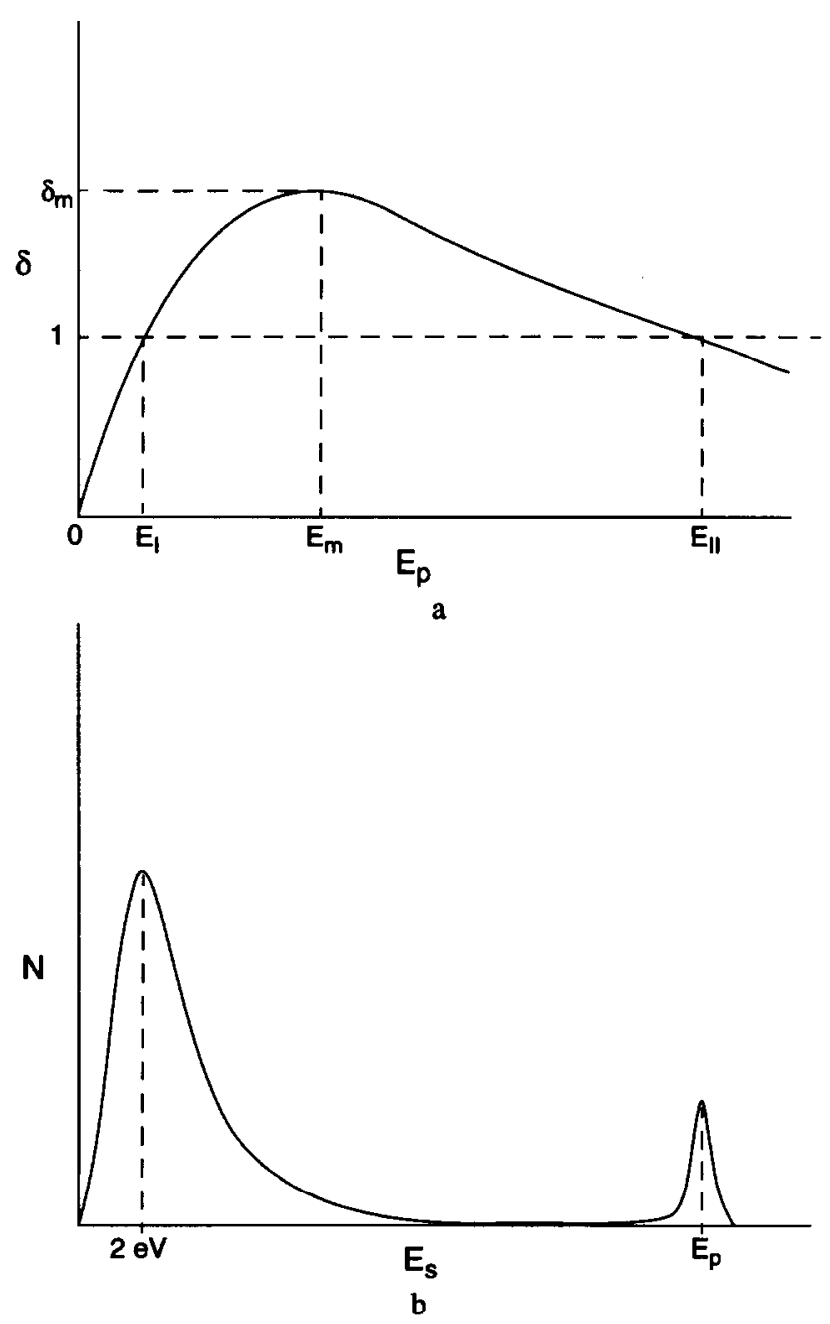

Fig. 1. (a) Typical example of the primary energy $\left(E_{\mathrm{p}}\right)$ dependence of the secondary electron emission yield $\delta$. For $\mathrm{MgO}$ one typically finds $E_{1}=20 \mathrm{eV}, E_{\mathrm{II}}=10 \mathrm{keV}, \delta_{\mathrm{m}}=12$ and $E_{\mathrm{m}}=950 \mathrm{eV}$. For metals typically $E_{\mathrm{I}}=80 \mathrm{eV}, E_{\mathrm{II}}=1 \mathrm{keV}, E_{\mathrm{m}}=400 \mathrm{eV}$ and $\delta_{\mathrm{m}}=1.5$ is obtained. (b) Typical example of the energy distribution of the secondary electrons.

loss of the primary electron $(-\mathrm{d} E / \mathrm{d} x)$ divided by the energy $\Omega$ required to produce one excited electron.

(2) The migration probability for an excited electron produced at depth $x$ to the surface is given by $\exp (-\alpha x)$, with $\alpha$ an effective inverse extinction length.

(3) The effective escape probability for a secondary electron that reaches the surface is $B$. 
(4) Angular scattering of the primaries is accounted for by the additional assumption that the average loss of energy is independent of the depth $x: \mathrm{d} E / \mathrm{d} x=-E_{\mathrm{p}} / R$, where $R$ is the penetration depth. This assumption is known as 'constant loss'.

(5) $R$ is determined by primary electrons which do not undergo angular scattering. In that case the energy loss of the primaries is governed by a power law: $\mathrm{d} E / \mathrm{d} x=-A / E^{n-1}$, where from experiments $n$ is found to be 1.3 to 1.6.

The secondary electron yield $\delta\left(E_{\mathrm{p}}\right)$ is now given by:

$$
\delta\left(E_{\mathrm{p}}\right)=B \times \frac{E_{\mathrm{p}}}{\Omega R} \times\left(1-\mathrm{e}^{-\alpha R}\right) / \alpha,
$$

i.e. the product of escape probability, density of excited electrons and an absorption term. From the power law (assumption (5)) it follows that the range $R$ and primary energy $E_{\mathrm{p}}$ are related by:

$$
R=\frac{E_{\mathrm{p}}^{n}}{A n}
$$

Experimentally it is found that $n \approx 1.35$ in the energy range 0.3 to $7 \mathrm{keV}[7,8]$.

The Inelastic Mean Free Path (IMFP) is a well-known empirical concept in Auger and XPS analysis, which shows a 'universal' energy dependence. For energies in the range 75 to $1000 \mathrm{eV}$ the IMFP is proportional to $E^{0.5}$ [9]. If we assume that the energy loss $-\mathrm{d} E / \mathrm{d} x$ is inversely proportional to the IMFP, then $n=1.5$ follows. However, the IMFP goes through a minimum at $E \approx 30 \mathrm{eV}$. This implies that $n$ will decrease when the primary energy is decreased below $75 \mathrm{eV}$.

When also taking into account the scattering of secondary electrons after their generation in the bulk, Dionne [10] derives the following expression for $E_{\mathrm{I}}\left(\right.$ valid for $\left.\delta_{\mathrm{m}}>1.5\right)$ :

$$
E_{\mathrm{I}}=0.51 E_{\mathrm{m}} \delta_{\mathrm{m}}^{-1.32},
$$

where $\delta_{\mathrm{m}}$ is the maximum secondary electron emission coefficient and $E_{\mathrm{m}}$ the primary energy at which this maximum is reached.

\section{Experimental}

Measurements of $\delta\left(E_{\mathrm{p}}\right)$ and $N\left(E_{\mathrm{s}}\right)$ are done in a UHV system at a base pressure of about $10^{-9} \mathrm{mbar}$. This UHV system is equipped with a Phi Cylindrical Mirror Analyser (CMA) and an Omicron 4-grid Retarding Field Analyser (RFA). 


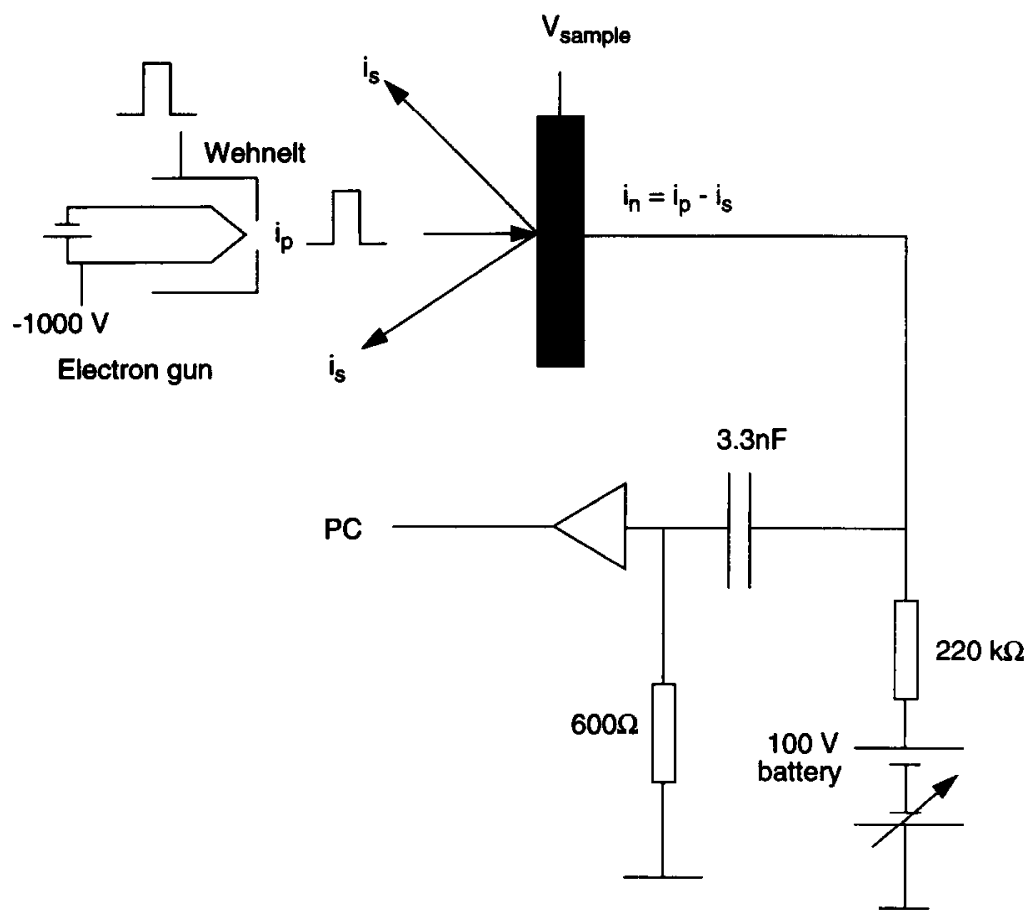

Fig. 2. Schematic overview of the experimental set-up.

To measure $\delta\left(E_{\mathrm{p}}\right)$ we use a measurement technique analogous to that of Henrich [11,12]. A schematic set-up is shown in Fig. 2. In this measurement the electron gun of the Phi CMA is used. The primary electron beam is pulsed to limit the amount of charge incident on the sample, in order to diminish the influence of charging on the measurement of $\delta\left(E_{\mathrm{p}}\right)$ of insulating samples. The pulse width is $5 \mu$ s and each pulse contains typically $10^{-12} \mathrm{C}$. The value of $\delta$ can be derived from the measured net current through the sample $i_{\mathrm{N}}$ :

$$
\delta=1-\frac{i_{\mathrm{N}}}{i_{\mathrm{p}}}
$$

The primary current $i_{\mathrm{p}}$ is determined by applying a potential of $+100 \mathrm{~V}$ to the sample. This pulls back most of the secondary electrons, so that $i_{\mathrm{p}} \approx i_{\mathrm{N}}$. Note that we include in $\delta$, measured in this way, both the 'true' secondary electrons and the inelastically and elastically scattered electrons. The value of $E_{\mathrm{p}}$ is varied by varying the negative potential of the sample, while keeping the cathode at a constant negative voltage. In the case of an insulating sample such a negative sample potential has the advantage that the measurement of $\delta\left(E_{\mathrm{p}}\right)$ is less influenced by charging [13]. 
To measure $N\left(E_{\mathrm{s}}\right)$ the sample is put in front of the 4-grids RFA. During the measurement of $N\left(E_{\mathrm{s}}\right)$ the two outermost grids are kept at earth potential, while the two middle grids are set to a retarding potential $V_{\mathrm{g}}$. When the kinetic energy of the secondary electrons is larger than $\left|\mathrm{e} V_{\mathrm{g}}\right|$ when they arrive at the first grid, they will be able to pass to the collector at $+300 \mathrm{~V}$. The current through the collector is subsequently measured as a function of $V_{\mathrm{g}}$. Numerical differentiation of this signal yields $N\left(E_{\mathrm{s}}\right)$. To increase the collection efficiency and to ease the use of low primary beam energies, a constant sample bias of $-50 \mathrm{~V}$ is used. This is a compromise between efficiency and resolution (the resolution is optimal when a field-free region exists between the sample and the first grid).

Samples which are used are a $100 \mathrm{~nm}$ e-beam evaporated $\mathrm{Au}$ layer on a $\mathrm{Si}$ substrate, $\mathrm{Si}$, stainless steel $(\mathrm{FeNiCr})$, leadglass $\left(\mathrm{PbO}+1 \% \mathrm{RuO}_{x}\right)$ and a $100 \mathrm{~nm}$ e-beam evaporated $\mathrm{MgO}$ layer on a Si substrate. All materials are measured 'as prepared', with the exception of the steel sample, which initially was severely contaminated by carbon and therefore directly sputter-cleaned. For $\mathrm{Si}$ this means that the data are representative for the native oxide, rather than for the semiconductor itself.

\section{Results and discussion}

\subsection{Universal yield curve}

In Fig. 3 we show for $\mathrm{Au}$, stainless steel, $\mathrm{Si}$, leadglass and $\mathrm{MgO}$ the reduced value $\delta_{\mathrm{c}} / \delta_{\mathrm{m}}$ as a function of $E_{\mathrm{p}} / E_{\mathrm{m}}$, in the following denoted as the 'reduced $\delta$-curve'. The energy scale is logarithmic, to emphasize the low-energy part. These data have been corrected for the contribution of elastic reflection, as will be discussed in Sec. 4.3. Figure 3 shows that the data for all materials follow more or less a single, 'universal', curve. For $E_{\mathrm{p}} / E_{\mathrm{m}}<1$, however, the spread in the reduced data is rather large, limiting the validity of the concept of a single 'universal' curve. The existence of a universal curve has also been mentioned by Baroody [14] and Dekker [3].

Also shown in this figure are curves representing the semi-empirical formula eq. (1), with $n=1.5,1.35$ and 1.05 , respectively. This formula describes the reduced data satisfactorily only if the exponent in the power law is allowed to vary from about 1.5 around $E_{\mathrm{p}} / E_{\mathrm{m}}=1$ to unity at the low-energy limit. This reduction of $n$, needed to fit the data properly at low primary energy, was already anticipated in Sec. 2 . At energies just above $E_{\mathrm{p}} / E_{\mathrm{m}}=1$, the value $n=1.35$ gives the best fit to the data; at higher energies the data show a convergence with the $n=1.5$ curve, in agreement with results reported in the literature [6-8]. The full curve in Fig. 3 is a Gaussian in the logarithm of $E_{\mathrm{p}} / E_{\mathrm{m}}$, 


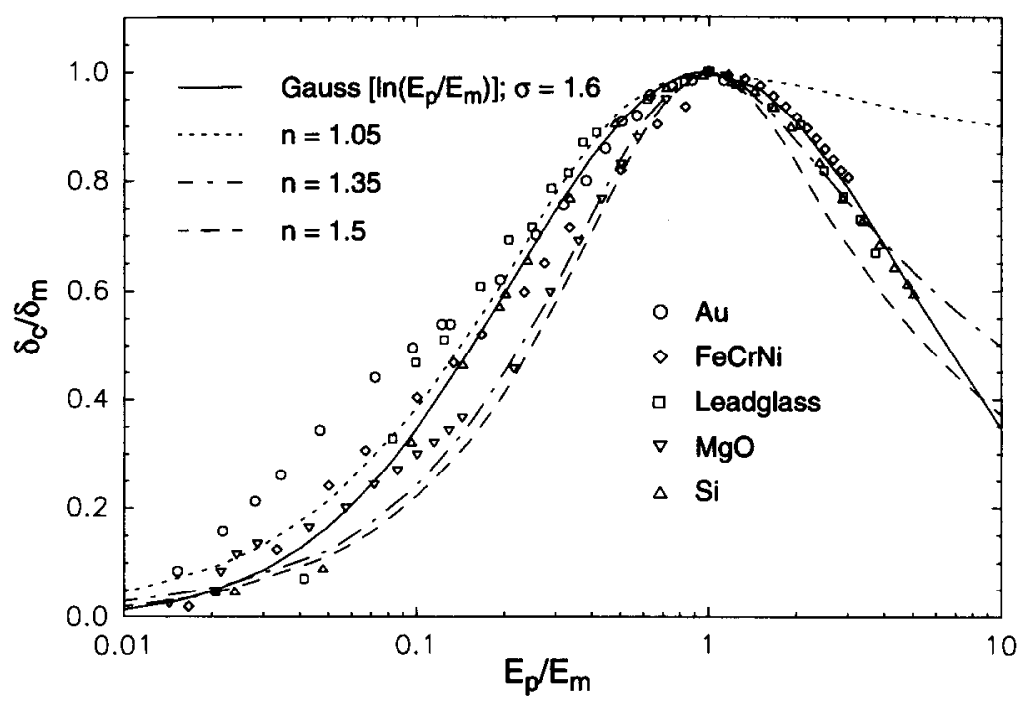

Fig. 3. Reduced $\delta$-curve for five materials, corrected for the elastic fraction. The fits are discussed in the text.

according to:

$$
\frac{\delta}{\delta_{\mathrm{m}}}=\exp \left\{-\frac{\left[\ln \frac{E_{\mathrm{p}}}{E_{\mathrm{m}}}\right]^{2}}{2 \sigma^{2}}\right\}
$$

with $\sigma=1.6$.

This single parameter curve describes the data quite well over the entire energy range, in fact much better than the semi-empirical formula for a fixed value of $n$. We found that even when omitting the correction for the elastic reflection, the fit of this equation to the data is still reasonable for $E_{\mathrm{p}}>100 \mathrm{eV}$. Although eq. (3) has no physical basis, it can be used in e.g. simulations of the measurement of the secondary electron emission of insulators [13] or in Monte Carlo simulations of the display [15].

\subsection{Estimate of the first crossover energy $E_{I}$}

The value of $E_{\mathrm{I}}$ can be estimated from the values of $\delta_{\mathrm{m}}$ and $E_{\mathrm{m}}$. First of all we have eq. (2) for $E_{\mathrm{I}}^{\mathrm{D}}$ derived by Dionne [10], and secondly, from eq. (3), the Gaussian fit to the reduced $\delta$-curve, we find for $E_{\mathrm{I}}$ :

$$
E_{\mathrm{I}}^{\mathrm{G}}=E_{\mathrm{m}} \exp \left(-\sqrt{5 \ln \delta_{\mathrm{m}}}\right) \text {. }
$$




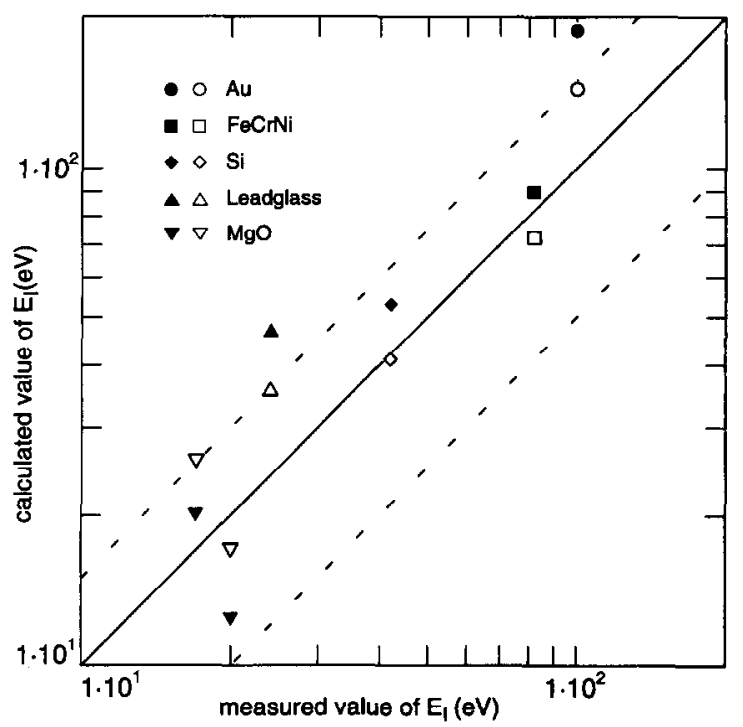

Fig. 4. Calculated vs measured value of $E_{\mathrm{I}}$. Open symbols represent $E_{\mathrm{I}}$ calculated using the Gaussian expression (eq. (4)). Filled symbols represent $E_{\mathrm{I}}$ calculated using the formula by Dionne (eq. (2)). Also shown is the line for which the calculated value equals the measured value of $E_{\mathrm{I}}$. Furthermore, a $50 \%$ error margin for the measured values of $E_{\mathbf{I}}$ (between the dashed lines) is depicted.

For $\mathrm{Au}, \mathrm{Si}$, steel, leadglass and $\mathrm{MgO}$ we have determined $E_{\mathrm{I}}, \delta_{\mathrm{m}}$ and $E_{\mathrm{m}}$. In Fig. 4 we show the calculated values $E_{\mathrm{I}}^{\mathrm{D}}\left(\delta_{\mathrm{m}}, E_{\mathrm{m}}\right)$ and $E_{\mathrm{I}}^{\mathrm{G}}\left(\delta_{\mathrm{m}}, E_{\mathrm{m}}\right)$ as a function of the measured $E_{\mathrm{I}}$. Although we find from Fig. 3 that the reduced $\delta$-curve can reasonably be approximated by a Gaussian expression (eq. (3)) and the expression by Dionne (eq. (1)), we find here that the theoretical estimates agree only within $50 \%$ of the measured value for $E_{\mathrm{I}}$. Probably this has to do with experimental artifacts encountered when measuring the secondary emission parameters. For insulators charging is the main cause for these artifacts, especially in the case of the measurement of $E_{1}$. An extensive study of experimental artifacts resulting from charging has been published elsewhere [13].

As the measurement of $E_{\mathrm{I}}$ is hampered more severely by charging than the measurement of $\delta_{\mathrm{m}}$ and $E_{\mathrm{m}}$, it is much easier to use the measurement of $E_{\mathrm{m}}$ and $\delta_{\mathrm{m}}$ for determining the suitability of an insulating coating for the use in the display than direct measurement of $E_{\mathrm{I}}$. The (apparent) $E_{\mathrm{I}}$ can then be determined using eq. (2) or eq. (4). Note that a suitable coating should have a small value for $E_{\mathrm{I}}$. From eq. (2) or eq. (4) it follows that this coating should, therefore, preferably have a large value for $\delta_{\mathrm{m}}$ combined with a small value for $E_{\mathrm{m}}$. 


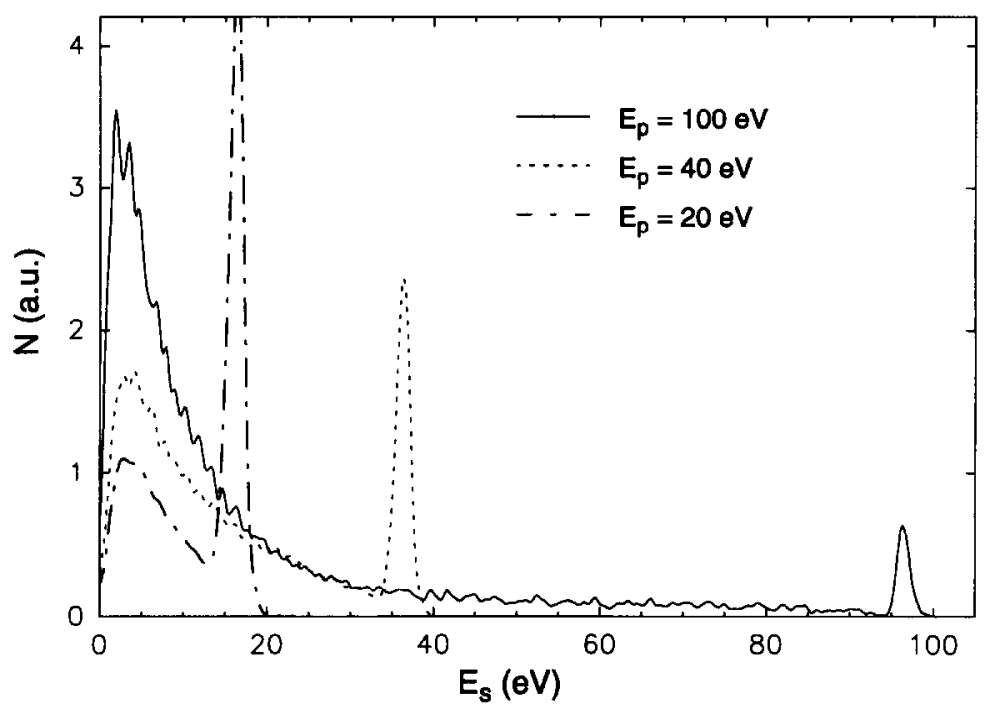

Fig. 5. Measured secondary electron energy distribution of $\mathrm{Au}$, for $E_{\mathrm{p}}=100,40$ and $20 \mathrm{eV}$.

\subsection{Energy distribution of secondary electrons}

Figure 5 shows the secondary electron energy distribution $N\left(E_{\mathrm{s}}\right)$ of $\mathrm{Au}$, for several primary energies. These curves are representative for all five materials. Clearly, the elastic peak is more prominent at lower primary energies. The instrumental resolution can be estimated from the falling edge of the elastic peak to be about $1.8 \mathrm{eV}$ FWHM. It can be seen that the elastic peaks are located $2.5 \mathrm{eV}$ below the nominal value, which is consistent with the contact potential difference between gun cathode $\left(\mathrm{LaB}_{6}\right)$ and $\mathrm{Au}$. We have shifted the $N\left(E_{\mathrm{s}}\right)$ distributions of the other materials, to make the onsets of the secondary peaks at $E_{\mathrm{p}}=100 \mathrm{eV}$ coincide with that of Au. This corrects for contact potential differences. The applied shifts were less than about $1 \mathrm{eV}$ in all cases.

In Fig. 6 we show the elastic fraction $f$, i.e. the integral of the elastic peak as a percentage of the total integral of $N\left(E_{\mathrm{s}}\right)$ as a function of primary energy, for all five materials. The data can be described by the following fit, as shown in the figure:

$$
\ln f=1.59+3.75 \ln E_{\mathrm{p}}-1.37\left(\ln E_{\mathrm{p}}\right)^{2}+0.12\left(\ln E_{\mathrm{p}}\right)^{3},
$$

with $E_{\mathrm{p}}$ in $\mathrm{eV}$ and valid for $E_{\mathrm{p}}$ values across the interval 5 to $100 \mathrm{eV}$. The relative increase of the elastic fraction towards lower primary energy was to be expected, since excitation of secondaries becomes increasingly unlikely, or 


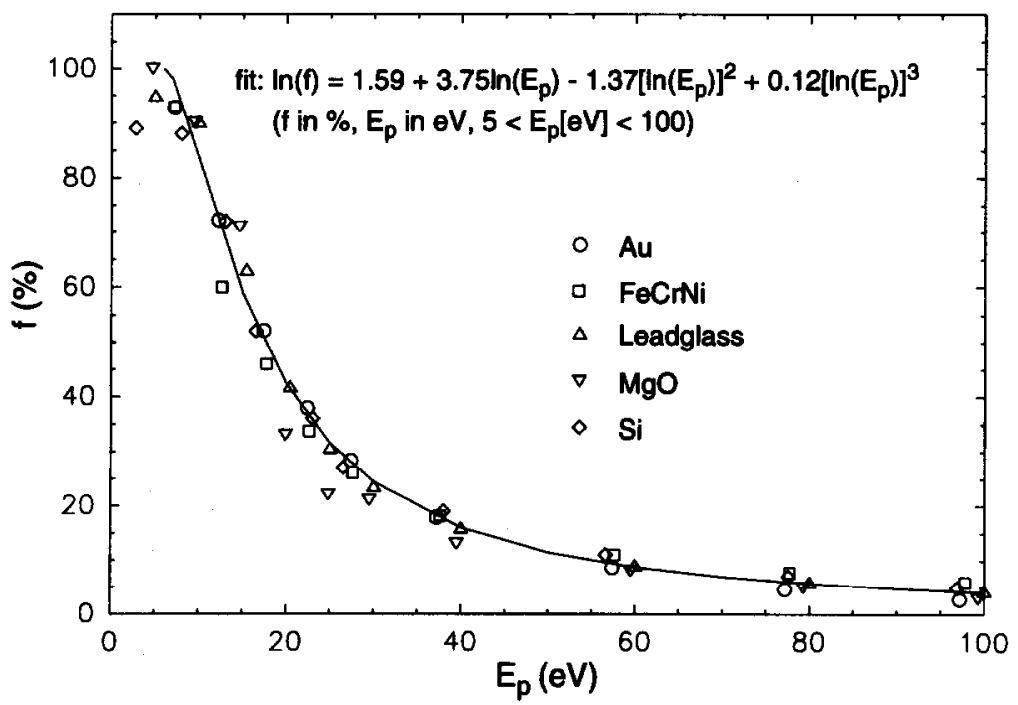

Fig. 6. Elastic fraction $f$ of the secondary electrons as a function of primary energy $E_{\mathrm{p}}$ for five different materials. The full line is the empirical fit as explained in the text.

even impossible for primary energies below approximately three times the bandgap energy [16]. However, the 'universal' shape of this curve is somewhat surprising and up to now we have not been able to find a satisfactory explanation.

We now define an elastic reflection coefficient $R$ :

$$
R \equiv \delta \times f,
$$

which is the number of elastically reflected electrons per incoming electron. The corrected secondary emission yield $\delta_{\mathrm{c}}$ is now determined as follows:

$$
\delta_{\mathrm{c}}=\frac{\delta-R}{1-R}=\delta \frac{1-f}{1-\delta f},
$$

which is the average number of secondary electrons which are inelastically scattered, $\delta-R$, divided by the number of actually penetrating electrons, $1-R$. This is the correction which is applied to the data in Fig. 3, since this is the quantity which is directly comparable with a theoretical yield curve. This correction is negligible above approximately $100 \mathrm{eV}$, but becomes increasingly important towards lower primary cnergy.

In Fig. 7 the low-energy part of the $N\left(E_{\mathrm{s}}\right)$ distribution is shown for $\mathrm{Au}$, $\mathrm{MgO}$, lead-glass and stainless steel. It is found that with decreasing $E_{\mathrm{p}}$ the maximum of the distribution shifts towards higher energy. This effect was present in all data, but most significant for the insulators. This behaviour 
was already predicted by Bindi et al. [17] by solving Boltzmann's equation. This effect can be understood intuitively by assuming that at a certain depth the primary electrons produce secondary electrons with an internal energy distribution which is independent of $E_{\mathrm{p}}$, having a maximum $E_{0}$ of a few $\mathrm{eV}$. While the secondaries travel towards the surface, their energy distribution changes. When the distribution is produced at a large depth (high $E_{\mathrm{p}}$ ), the probability for inelastic scattering increases and therefore $E_{0}$ is expected to shift to a lower energy with increasing $E_{\mathrm{p}}$. The fact that this is more pronounced in the case of insulators is more difficult to explain. One would, on the contrary, expect that for insulators this effect should be less pronounced, as secondary electrons are expected to have a larger mean free path due to the absence of free-electron scattering and because of the large band gap which makes it unlikely for secondary electrons with a kinetic energy of approximately three times the band gap to interact with valence electrons [16]. Note that for insulators also charging can play a role in determining the shape of the energy distribution. The influence of charging is unknown, however.

The low-energy part of the distributions has been fitted to a MaxwellBoltzmann distribution:

$$
f^{\mathrm{MB}}\left(E_{\mathrm{s}}\right)=C_{1} \frac{E_{\mathrm{s}}}{E_{0}} \exp \left(-\frac{E_{\mathrm{s}}}{E_{0}}\right) .
$$

This distribution is a simplified version (containing less fit parameters) of the one derived by Bouchard and Carette [18], and turned out to be sufficient for use in Monte Carlo simulations [15]. A slightly more complicated formula, giving better fit results, is a Gaussian with a logarithmic argument:

$$
f^{\mathrm{G}}\left(E_{\mathrm{s}}\right)=C_{2} \exp \left\{-\frac{\left[\ln \frac{E_{\mathrm{s}}}{E_{0}}\right]^{2}}{2 \tau^{2}}\right\} \text {. }
$$

In both formulas $C_{i}$ is the normalization constant and $E_{0}$ is the position of the maximum. The Gaussian curve has the standard deviation $\tau$ as an additional parameter. Note that the average secondary electron energy $E_{\mathrm{a}}$ is given by:

$$
E_{\mathrm{a}}^{\mathrm{MB}}=2 E_{0}, \quad E_{\mathrm{a}}^{\mathrm{G}}=E_{0} \exp \left(1.5 \tau^{2}\right),
$$

for eq. (5) and (6), respectively. Fit results for the materials shown in Fig. 7 are given in Table I. Note that these fits actually represent convolutions of the above formulas with a Gaussian resolution function with $\tau=0.8 \mathrm{eV}$. This resolution function affects the rising edge and the position of the maximum significantly. From Table $\mathrm{I}$ it can be concluded that the variations in $\tau$ are 


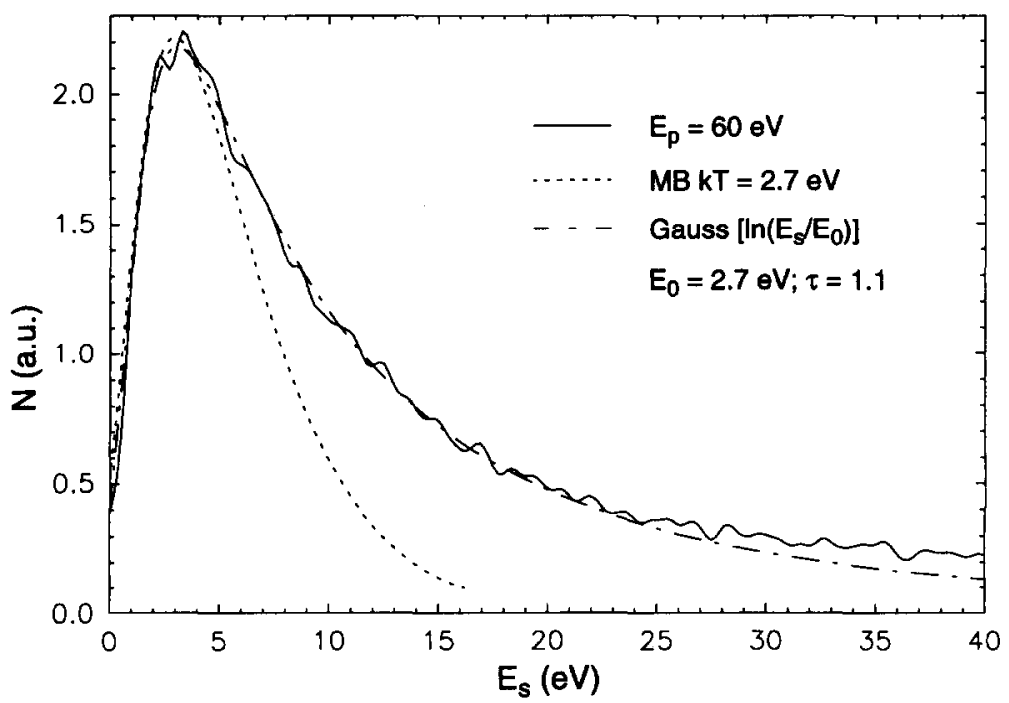

a

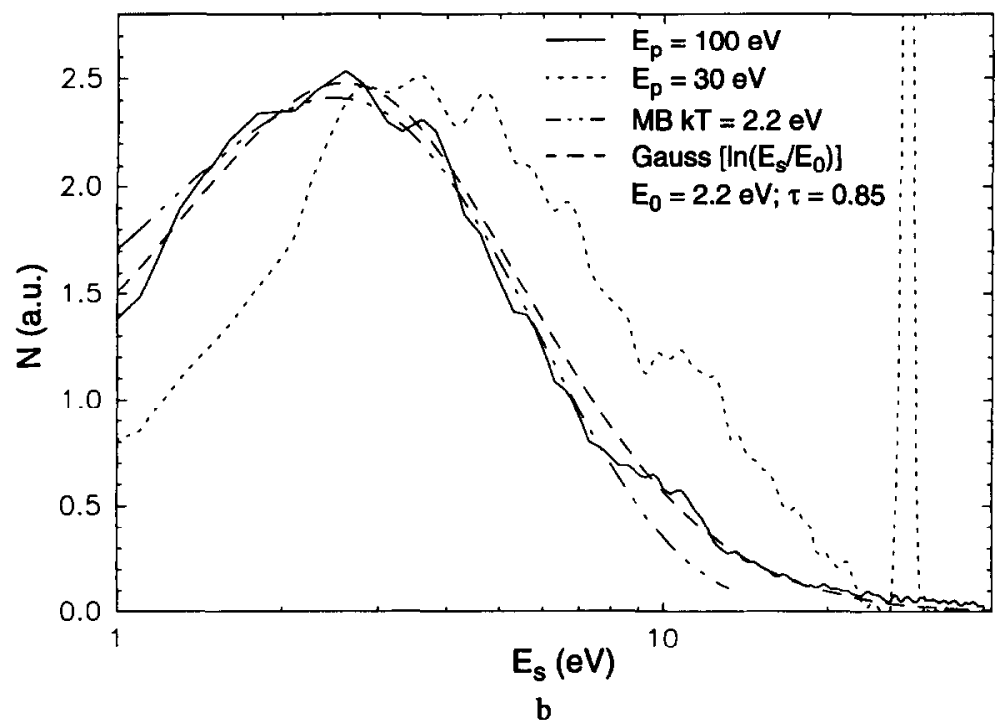

Fig. 7. Low-energy part of the secondary electron energy distributions for several materials. Some of the curves have been fitted to a Maxwell-Boltzmann distribution and a Gaussian. The fit parameters are shown in Table I. (a) Au, fitted is the curve for $E_{\mathrm{p}}=30 \mathrm{eV}$; (b) $\mathrm{MgO}$, fitted is the curve for $E_{\mathrm{p}}=100 \mathrm{eV}$. 


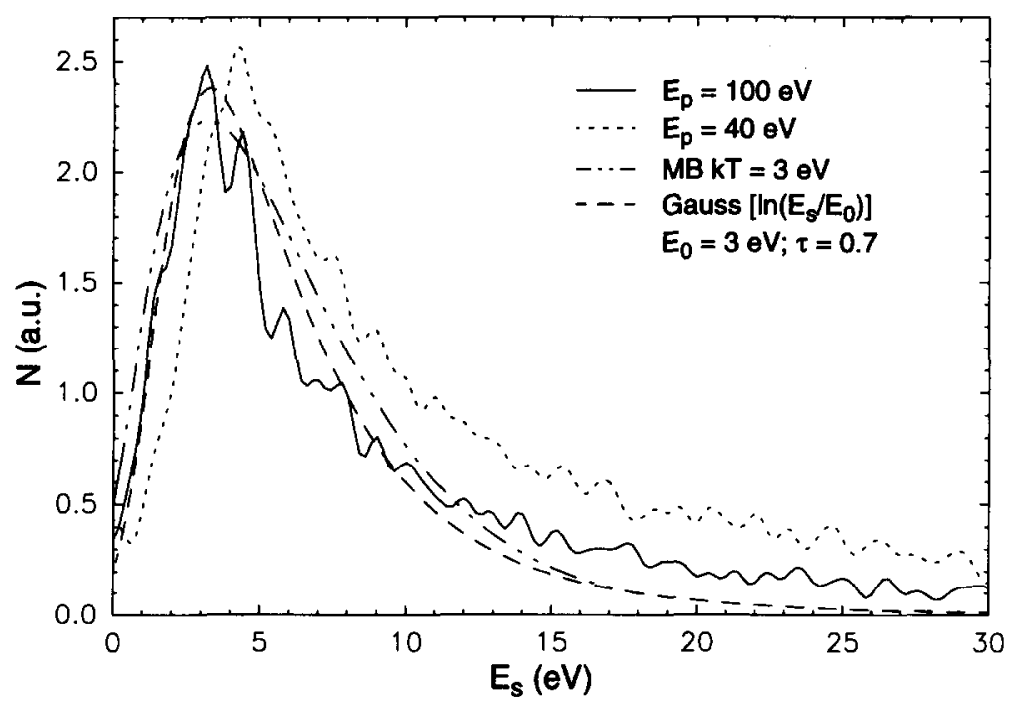

c

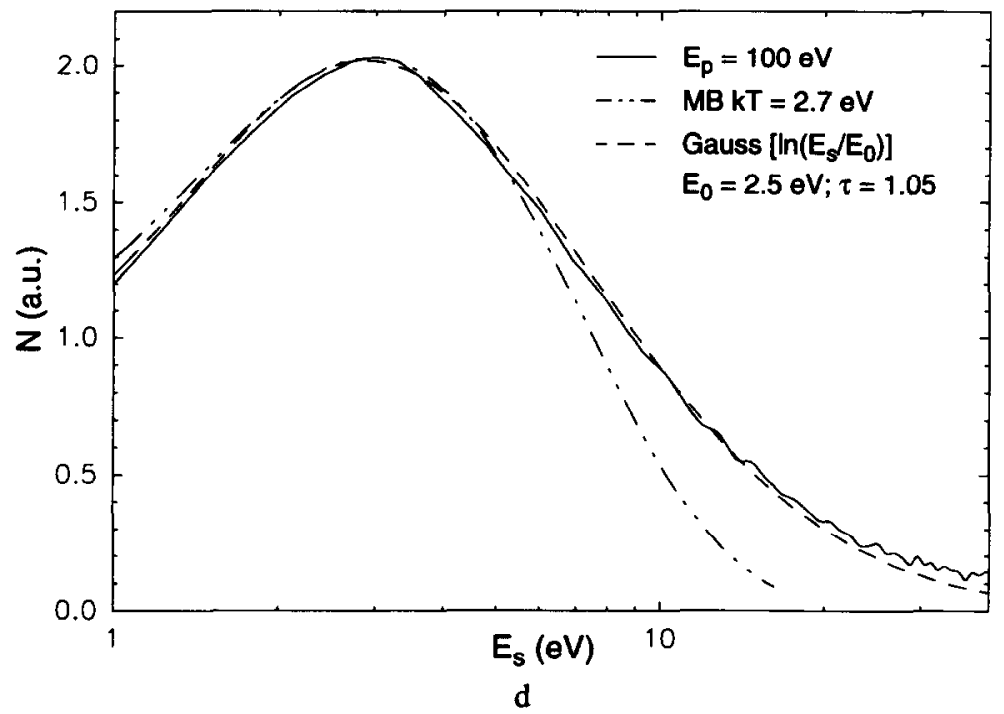

Fig. 7. contd; (c) Leadglass, fitted is the curve for $E_{\mathrm{p}}=100 \mathrm{eV}$; (d) Stainless steel. 
TABLE I

Overview of the fit parameters used in the fits of Fig. 7.

\begin{tabular}{lccc}
\hline Materials & $E_{0}(\mathrm{eV})$ & $\tau(\mathrm{eV})$ & $E_{\mathrm{a}}^{\mathrm{G}}$ \\
\hline Au (fit to $\left.E_{\mathrm{p}}=60 \mathrm{eV}\right)$ & 2.7 & 1.1 & $6.1 E_{0}$ \\
$\mathrm{MgO}$ (fit to $\left.E_{\mathrm{p}}=100 \mathrm{eV}\right)$ & 2.2 & 0.85 & $3.0 E_{0}$ \\
Leadglass (fit to $\left.E_{\mathrm{p}}=100 \mathrm{eV}\right)$ & 3.0 & 0.7 & $2.1 E_{0}$ \\
Stainless steel (fit to $\left.E_{\mathrm{p}}=100 \mathrm{eV}\right)$ & 2.7 & 1.05 & $5.2 E_{0}$ \\
Si (fit to $\left.E_{\mathrm{p}}=100 \mathrm{eV}\right)$ & 2.0 & 1.1 & $6.1 E_{0}$ \\
\hline
\end{tabular}

significant which implies that a Gaussian fit, which decouples the width of the distribution and the position of the maximum, is preferable.

\section{Conclusions}

It is experimentally shown that for the materials studied in this paper the reduced yield $\delta / \delta_{\mathrm{m}}$ as a function of the reduced energy $E_{\mathrm{p}} / E_{\mathrm{m}}$ shows a more or less universal behaviour. When using the semi-empirical formula by Dionne to fit the data for various values of $n$, we find that the best fit to the high-energy part of the reduced $\delta$-curve is the fit using $n=1.5$, while the best fit to the low-energy part is the fit using a lower value for $n$. This result can be understood from the energy dependence of the inelastic mean free path. We found that a Gaussian with a logarithmic argument with $\sigma=1.6$ describes the reduced $\delta$-curve better than the semi-empirical formula of Dionne. It is concluded that $E_{\mathrm{m}}$ and $\delta_{\mathrm{m}}$ can be used qualitatively as a measure for the suitability of a coating for use in the display.

We also found that the energy distribution of the secondary electrons can accurately be described by a Gaussian with a logarithmic argument, which can be used in Monte Carlo simulations. Furthermore, we found that the elastic fraction of the secondary electrons exhibits a surprisingly 'universal' behaviour. At present no satisfactory explanation is available, however.

\section{Acknowledgements}

We would like to thank G. van Gorkom, S. de Zwart, B. Hendriks and N. Lambert for useful discussions. 


\section{REFERENCES}

[1] G.G.P. van Gorkom, Introduction to Zeus displays, Philips J. Res., this issue (1996).

[2] S.T. de Zwart, G.G.P. van Gorkom, B.H.W. Hendriks, N. Lambert and P.H.F. Trompenaars, Basics of electron transport over insulators, Philips J. Res., this issue (1996).

[3] A.J. Dekker, Solid State Physics, F. Seitz and D. Turnbull, eds, Academic Press, New York, 1958 , Vol. 6, p. 271.

[4] V.E. Henrich and J.C.C. Fan, Appl. Phys. Lett. 23 (1), 7 (1973).

[5] N.R. Whetten and A.B. Laponsky, J. Appl. Phys. 30, 432 (1959).

[6] G.F. Dionne, J. Appl. Phys. 44, 5361 (1973).

[7] J.R. Young, J. Appl. Phys. 27, 1 (1956).

[8] J.R. Young, J. Appl. Phys. 28, 524 (1957).

[9] M.P. Seah and W.A. Dench, Surface and Interface Analysis 1, 2 (1979).

[10] G.F. Dionne, J. Appl. Phys. 46, 3347 (1975).

[11] V.E. Henrich, Rev. Sci. Instrum. 44 (4), 456 (1973).

[12] V.E. Henrich, Rev. Sci. Instrum. 45(6), 861 (1974).

[13] J.J. Scholtz, R.W.A. Schmitz, B.H.W. Hendriks and S.T. de Zwart, Proceedings IVESC '96, to be published in Appl. Surf. Sci.

[14] E.M. Baroody, Phys. Rev. 78, 780 (1950).

[15] B.H.W. Hendriks, G.G.P. van Gorkom, A.T.M.H. van Keersop, N. Lambert, P.H.F. Trompenaars and S.T. de Zwart, Monte Carlo calculations of the electron hop transport in various parts of a Zeus display, Philips J. Res., this issue (1996).

[16] R.C. Alig and S. Bloom, J. Appl. Phys. 49, 3476 (1978).

[17] R. Bindi, H. Lanteri and P. Rostaing, J. Phys. D: Appl. Phys. 13, 267 (1980).

[18] C. Bouchard and J.D. Carette, Surf. Sci. 100, 251 (1980). 